\title{
Image fidelity improvement in digital holographic microscopy using optical phase conjugation
}

\author{
Huang-Tian Chana, Yang-Kun Chew ${ }^{b}$, Min-Tzung Shiuc, and Chi-Ching Changa,* \\ ${ }^{a}$ Department of Materials and Energy Engineering, MingDao University, Taiwan \\ ${ }^{b}$ Optoelectronics Research Centre, University of Southampton, United Kingdom \\ ${ }^{c}$ School of Defense Science, National Defense University, Taiwan
}

\section{ARTICLE INFO}

Article history:

Received:

Keywords:

Digital holographic microscopy

Self-pumped phase conjugation

Photorefractive

Noise suppression

Phase Retrieval

\begin{abstract}
With respect to digital holography, techniques in suppressing noises derived from reference arm are maturely developed. However, techniques for the object counterpart are not being well developed. Optical phase conjugation technique was believed to be a promising method for this interest. $\mathrm{A} 0^{\circ}$-cut $\mathrm{BaTiO}_{3}$ photorefractive crystal was involved in self-pumped phase conjugation scheme, and was employed to in-line digital holographic microscopy, in both transmission-type and reflection-type configuration. On pure physical compensation basis, results revealed that the image fidelity was improved substantially with 2.9096 times decrease in noise level and 3.5486 times increase in the ability to discriminate noise on average, by suppressing the scattering noise prior to recording stage.
\end{abstract}

\section{Introduction}

Digital holography (DH), wherein interference patterns are captured using charge-coupled devices (CCD), was pioneered by Schnars and Jüptner in 1994 [1]. They used numerical reconstruction of optical wavefronts to retrieve information of objects, which are fundamental to DH. DH has not only improved the image quality of a conventional microscopy with extended depth of focus, but also conferred considerable advantages over physical methods, particularly time saving (avoiding chemical developing procedures). With computational tools, both zero-order (DC) and first-order conjugate (twin image) noise term can be suppressed numerically, such as angular spectrum filtering [2], speckle method [3], and phase shifting [4-6] techniques. Nevertheless, these profound techniques only suppress noises that are derived from reference arm; the object arm, on the other hand, is not being paid with equivalent attention.

Noises contributed by hardware and electronics [7, 8] are significant. Nevertheless, scattering due to optical elements, dust particles or even the object of interest itself is quite inevitable; if the object signal itself associates terrible noises, those noises will of course be reconstructed alongside with. In terms of suppressing scattering light, phase modulation [9], coherence modulation [10], polarization modulation [11, 12], intensity modulation [13], frequency modulation [14] and correlation algorithms [15] are common techniques. However, additional optical elements are feasible but may also be potential sources of scattering noise; hence, these techniques can only suppress scattering noise to a certain extent. Notwithstanding, there is another candidate that had been active from 1980 2000, possess huge potential in suppressing scattering noise, i.e. phase conjugation technique. It is a unique technique that enables back propagation of wave to its original state. This property is perfect for suppressing scattering noise - in an ideal scenario, if a scattered signal is traced back to a point just before it scatters, no scattering noises will present in the signal.

There are of course many methods available in performing phase conjugation; in digital holographic microscopy (DHM), new phase conjugation technique has been reported, namely digital optical phase conjugation [16, 17], was used to enhance the signal quality by suppressing turbidity of the object. Meanwhile, phase conjugation involving non-linear optics has also been reported; however, the photorefractive crystals were used as a medium to record hologram $[18,19]$, extracting conjugated wave from the negative first-order diffraction term. The photorefractive crystals were simply utilized for their dynamic properties of rewriting different holograms for different objects; however, writing and reading holograms (producing phase-conjugated wave) were performed separately. Nonetheless, these endeavors showed great improvement in the quality of reconstructed images by undoing scattering noise using phase conjugation techniques, prior to recoding stage. Thus, it should be clear that suppression of noises from the object arm prior to the recording stage plays a significant role. 
With respect to phase conjugation using photorefractive crystals, four-wave mixing is commonly used [20]. With our empirical knowledge upon optical phase conjugation (OPC) [21, 22], we determined that OPC via photorefractive crystals suppresses scattering noise but intrinsically does not have a high spatial resolution and fails to compensate for relatively high spatial frequency signals due to its dimension. By contrast, microscopy enhances both the resolution and scattering. These two techniques appear to have a complementary relationship, and their combination can be optimized to obtain a favorable result. Furthermore, $\mathrm{BaTiO}_{3}$ photorefractive crystal is one of the promising choices that inherit excellent coupling characteristics [23]. Thence, this paper presents a novel application that utilizes $\mathrm{BaTiO}_{3}$ photorefractive crystal for self-pumped phase conjugation (SPPC) [24] in DHM (SPPCDHM) to suppress scattering noise prior to recording stage. Different from previous methods, the combinations of gratings formed within the photorefractive crystal are being simultaneously read and written, enabling near real time phase conjugation.

\section{Theoretical background}

\section{A. Phase conjugation}

In order to show that how phase conjugation technique suppresses scattering noises prior to recording stage, a qualitative illustration is shown in Fig. 1. A plane wave carried twice the phase delay introduced by the phase object using a conventional mirror (CM), whilst phase-conjugated mirror (PCM) on the other hand, showed that the reflected signal is retraced to its initial form. The illustration of course can be expended to arbitrary wavefront rather than just plane wave. Hence, by using a PCM, those double-passed elements were as if they did not exist; thus, noises were suppressed.

Since the return signal is phase conjugated, an inversion of sign with respect to phase distribution is expected. The return complex amplitude of that using CM and PCM is expressed in Eq. 1 and Eq. 2, respectively, for the case of using phase shifting technique.

$$
\begin{aligned}
& H_{1, C M}^{\prime}-H_{2, C M}^{\prime}\left(e^{-i \Delta \phi}\right)=\psi_{O} \psi_{R}^{*}\left(1-e^{-i 2 \Delta \phi}\right), \\
& H_{1, P C M}^{\prime}-H_{2, P C M}^{\prime}\left(e^{-i \Delta \phi}\right)=\psi_{O}^{*} \psi_{R}^{*}\left(1-e^{-i 2 \Delta \phi}\right) .
\end{aligned}
$$

$H_{1}^{\prime}$ and $H_{2}^{\prime}$ are holograms before and after phase modulation respectively, which were subjected to zero-order noise suppression. $\psi_{O}$ and $\psi_{R}$ denotes the object wave and reference wave, whilst $\Delta \phi$ represents the phase modulation respectively. $\psi_{R}$ is assumed to be a plane wave, and can be treated as a plane wave in our case [25]. The asterisk sign $\left({ }^{*}\right)$ denotes complex conjugate of the wavefront. Hence, it is possible to reconstruct images using only free space propagation to show intrinsic improvement in image fidelity.

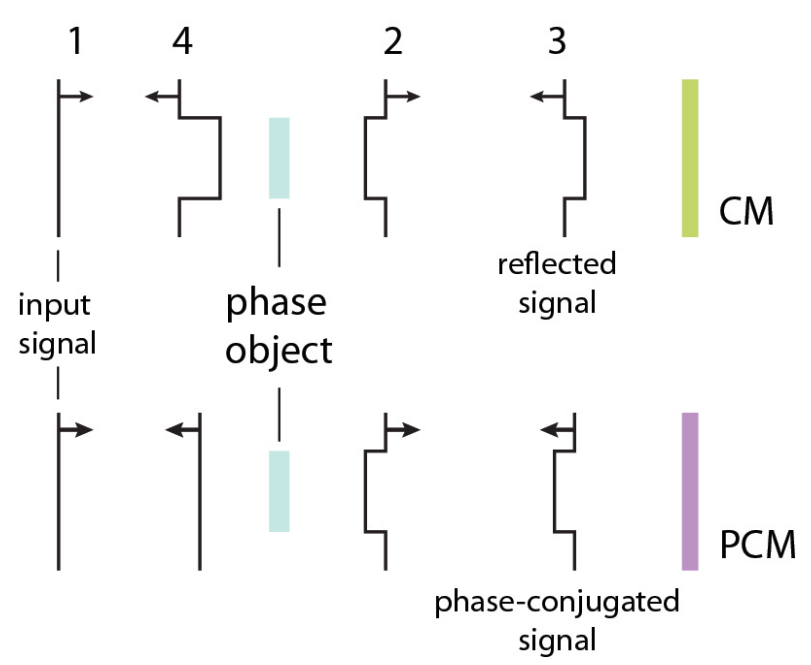

Fig. 1. Schematic of noise suppression using optical phase conjugation technique.

\section{B. Measure of image fidelity}

\section{B1. Signal-to-noise-ratio}

In order to show quantitative gain of the capacity of phase conjugation technique in suppressing noises, signal-to-noise ratio (SNR) of reconstructed images is evaluated. To calculate SNR, a reference signal is required. In terms of intensity, a reference signal can be easily obtained; however, the phase counterpart that requires numerical method would be less objective. Nevertheless, since DHM is very sensitive to phase perturbation, any suppression of noises would be distinctive even with qualitative comparison. Hence, the discussion of SNR will be limited to the aspect of intensity only. Let the image (without interference) recorded at the image plane to serve as the reference basis $A(x, y)$, while the same image of that subjected to externally added noise via a transparency will serve as the test image $B(x, y)$. Consider the recorded images have size of $M \times N$, the evaluation method for SNR can be expressed as

$$
S N R_{A, B}=10 \log _{10}\left(\frac{\sum_{x=1}^{N} \sum_{y=1}^{M}|A(x, y)|^{2}}{\sum_{x=1}^{N} \sum_{y=1}^{M}|A(x, y)-B(x, y)|^{2}}\right) .
$$

The concept of SNR may be a bit vague in here. So, instead of decibels, the obtained figures are translated into weightage, $W$, which is of course equals to noise/(noise + signal). Noise is obtained from the denominator of SNR; likewise, signal is obtained from the numerator.

$W=\frac{\text { Noise }}{\text { Noise }+ \text { Signal }}$.

\section{B2. Discrimination of noise}

SNR provides us the noise level associated with the signal of interest. It is however that we have no confidence in differentiating noise and signal from one and the other. 
Thus, a new measuring criterion should be introduced. Since the added noise were mostly due to (but not limited to) scattering, which has lower intensity (true in general); thus, using intensity thresholding to discriminate (distinguish) signal and noise would be reasonable. The threshold value would then indicates that a signal is required to have intensity greater than the threshold value (relative to the max intensity), in order to be considered as signal, but not noise. Therefore, the higher the threshold value, one can discriminate noise from the signal of interest with greater confidence.

$$
\begin{aligned}
T_{C M} & =\frac{(1-W)}{3 \cdot\left\langle W_{C M} / W_{P C M}\right\rangle} . \\
T_{P C M} & =\frac{(1-W)}{3} .
\end{aligned}
$$

The threshold value, $T$ is defined to be the average difference between overall signal and percentage of noise. Rather than 2, the factor of 3 is chosen to allow a window of tolerance. For the case of using CM, another weighting factor is used for normalization to the case accounted for using PCM. The weighting factor is the average ratio of noise level of that using $\mathrm{CM}$ and PCM, with respect to transmission- and reflection-type. It should be reminded that because the results were compared in different experiment configuration, and with different reference basis (non-ideal basis). Therefore, the method of evaluation is quite unusual to be seen.

\section{Experiment}

SPPCDHM is a modified configuration based on Mach-Zehnder setup, as shown in Fig. 2. A 532 nm DPSS Verdi laser was used as a light source. A half-wave plate (HWP) and polarizer were used to produce an extraordinary beam. A variable beam splitter (VBS) was used to adjust the intensity ratio of the object and reference wave. A long focal condenser lens was used to focus on the object of interest, such that the area of interest was within the depth of focus, probed by plane wave, which in turn reduces scattering. The object (USAF 1951, negative target, Newport) was then magnified by a microscopic objective (MO, $\mathrm{M}=20 \mathrm{x}, \mathrm{NA}=0.40$ ). A lens was used to retrieve the magnified image and focus into the $0^{\circ}$-cut $\mathrm{BaTiO}_{3}$ photorefractive crystal, such that relatively high spatial frequency signals could be compensated through OPC. A spatial filter (SF) in the reference arm was used to match the quadratic phase term associated with the object beam [25]. A glass plate with a thickness of $177 \mu \mathrm{m}$ and refractive index of 1.52 was placed in the reference arm as phase object for arbitrary micro-phase shifting technique [26]. The object and reference beam were merged using a beam splitter (BS), and the hologram was recorded by a CCD (Pixera-150SS, $1040 \times 1392$ pixels, $0.484 \times 0.650 \mathrm{~cm}^{2}$ ).

Knowing that SPPCDHM is a robust architect, apart from ordinary reconstruction procedure, a $0.095 \mathrm{~mm}$ thick transparency (for color printing) was placed between the lens and PCM/CM in the object arm, to imitate scattering of optical elements. The noise added hologram was then subjected to recording and reconstruction to investigate the performance of SPPCDHM. The whole experiment was repeated by using a CM in place of PCM. The reconstructed images, both intensity and phase distribution were shown in Fig. 3. Since the object has amplitude discontinuity, the reconstructed phase distribution will gain artificial fluctuations in the blocked (undefined) region. Thus, a binary mask, derived from the reconstructed intensity with certain threshold value, was applied to the phase distribution. The results were perfectly in agreement with our predictions, such that the phase flipped signs but remain the same in magnitude $(+1.5 \mathrm{rad}$. to $-1.5 \mathrm{rad}$. on average). Furthermore, SPPCDHM is able to suppress scattering noise as well as compensate phase aberrations that were introduced by probing the transparency with converging spherical wave.

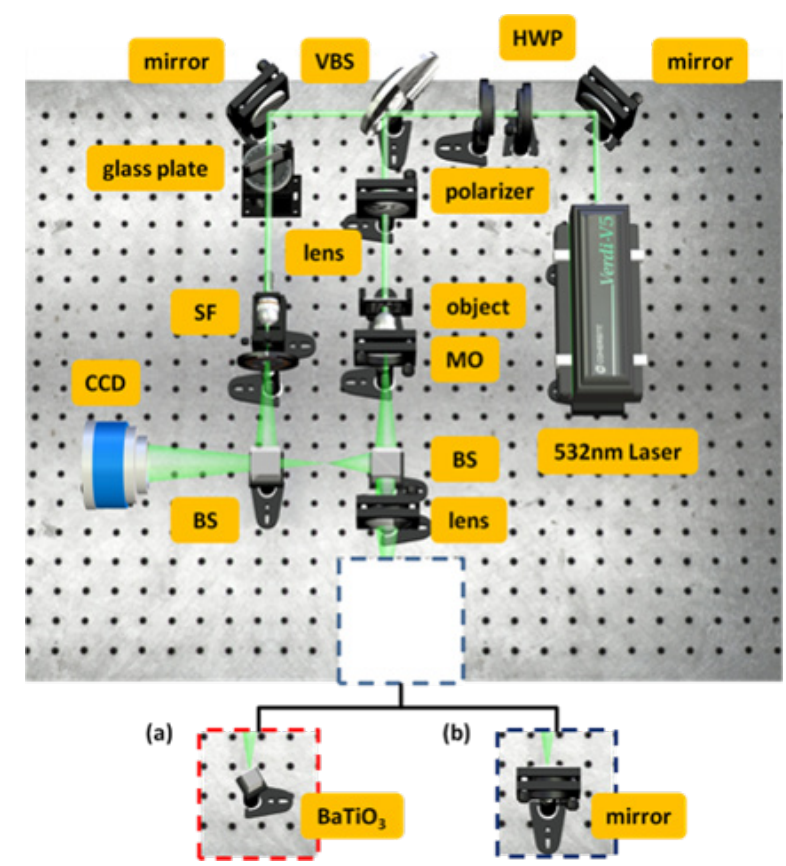

Fig. 2. Transmission-type SPPCDHM. Manipulated optical elements are delineated by dotted-line boxes: (a) a $0^{\circ}$-cut $\mathrm{BaTiO}_{3}$ photorefractive crystal as PCM, and (b) control set with the use of CM.

As we can see the capabilities of noise suppression of SPPCDHM in transmission-type, we further introduce it unto reflection-type DHM. The reference arm will be the same as transmission-type, adaptive to the object arm. The object arm on the other hand, was comprised of a SF, lens and MO, forming a tele-centric configuration to probe the object of interest (USAF 1951, positive target, Edmund Optics) with plane wave. A polarized beam splitter (PBS) and a quarter-wave plate (QWP) were used to minimize intensity loss of object signal. The object signal was p-polarized for a better coupling effect via a HWP. The object signal was then focused into a PCM and directed towards the CCD; the reflection-type SPPCDHM architect as shown in Fig. 4. The same procedures were conducted; results were shown in Fig. 5. 
(a)

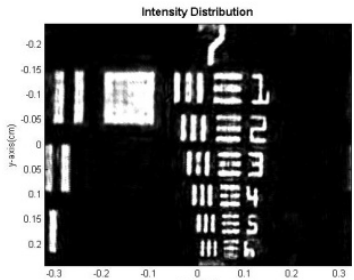

(e)

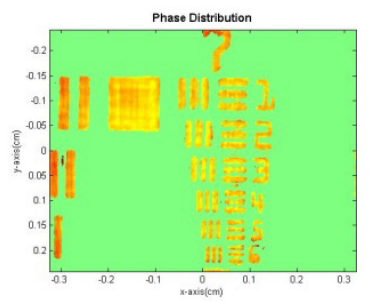

(b)

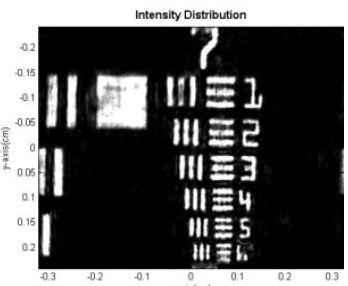

(f)

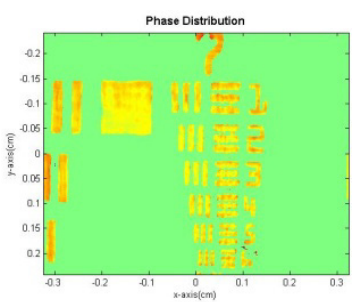

(c)

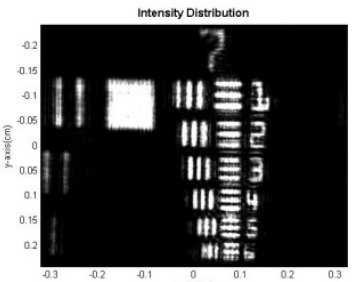

(g)

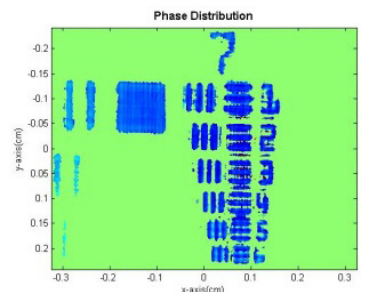

(d)

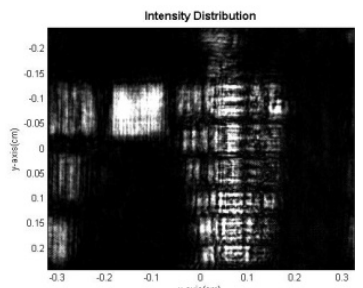

(h)

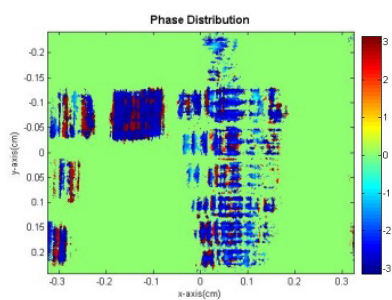

Fig. 3. (a-d) Reconstructed images of that using SPPCDHM and its control set under transmission-type configuration, without and with transparency introduced respectively, while (e-h) are corresponding phase distribution after binary mask filter.

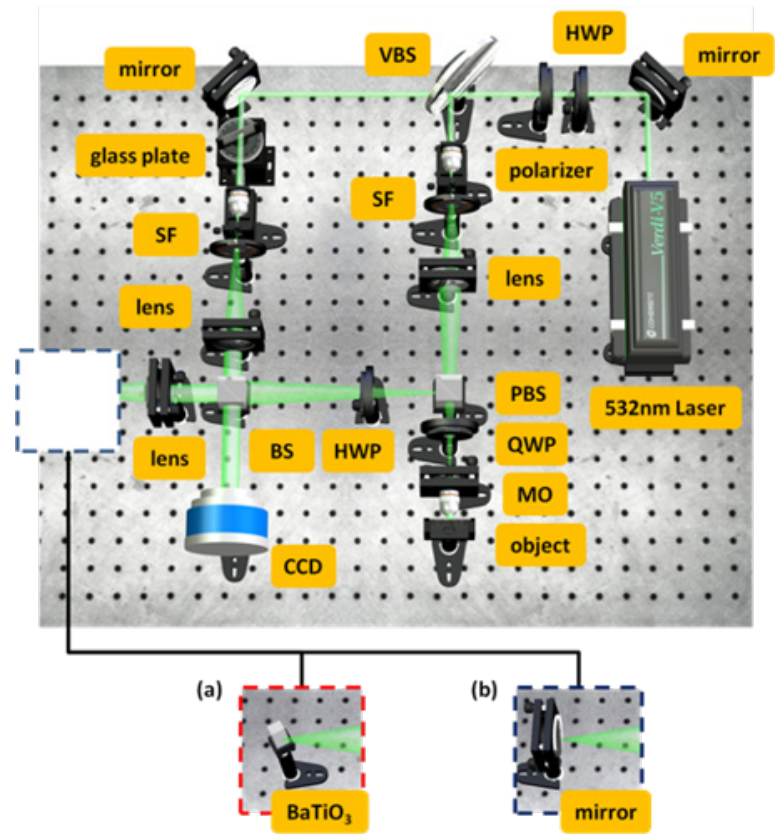

Fig. 4. Reflection-type SPPCDHM. Manipulated optical elements are delineated by dotted-line boxes: (a) a $0^{\circ}$-cut $\mathrm{BaTiO}_{3}$ photorefractive crystal as PCM, and (b) control set with the use of CM.

First of all, before any reconstruction procedure being taken place, the SNR for each configuration is computed (as shown in Table 1) by comparing intensity profile of images at image plane (without interference), of that with and without the transparency added. The calculated values will then be translated into weightage of noise level associated, as shown in Table 2. From Table 2, the difference of weightage in terms of ratio is noted to be $\sim 4$ and $\sim 2$. So, the extra weighting factor for our case would be 2.9096 . The weightage will then help us to determine the threshold value for generating the binary filter mask for the reconstructed phase distribution, as shown in Table 3. Subsequently, hologram was recorded, and image was reconstructed using Huygen's convolution method only, without any other numerical techniques. The binary mask is then derived from respective reconstructed intensity distribution with the appropriate threshold value computed in advance. The binary mask is applied to respective phase distribution only, in order to get rid of the artificial fluctuations due to amplitude discontinuity. In addition, the filtered phase distribution will show the level of confidence for SPPCDHM in discriminating noise; in turn, validates that the method of evaluation used is appropriate. As stated in previous section, SNR was calculated with respect to intensity only. Yet, by subjecting the phase distribution to binary mask filter, a qualitative contrast can be visualized easily. Despite of being qualitative, the comparison provides more direct insights. Indeed, further increase in threshold value will corrupt the reconstructed images.

Table 1. Image fidelity of reconstructed images.

\begin{tabular}{|c|c|c|}
\hline \multirow{2}{*}{} & \multicolumn{2}{|c|}{$\operatorname{SNR}_{A, B}(\mathrm{~dB})$} \\
\cline { 2 - 3 } & SPPCDHM & Control Set \\
\hline Transmission-type & 11.2630 & 3.7961 \\
\hline Reflection-type & 8.6541 & 6.2861 \\
\hline
\end{tabular}

Table 2. Associated noise level in retrieved signal.

\begin{tabular}{|c|c|c|}
\hline \multirow{2}{*}{} & \multicolumn{2}{|c|}{ Associated noise (\%) } \\
\cline { 2 - 3 } & SPPCDHM & Control Set \\
\hline Transmission-type & 6.9564 & 29.4406 \\
\hline Reflection-type & 11.9973 & 19.0397 \\
\hline
\end{tabular}


Without transparency

(a)

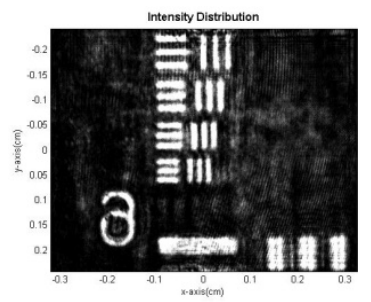

(e)

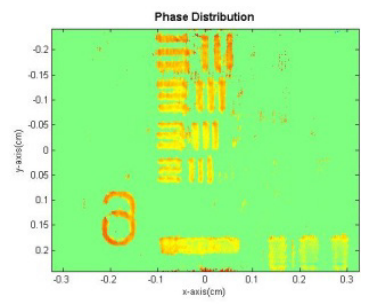

With transparency

(b)

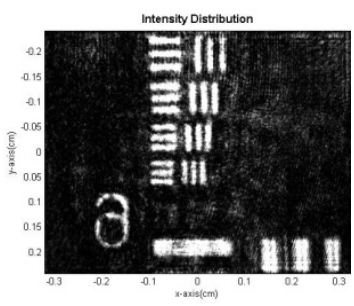

(f)

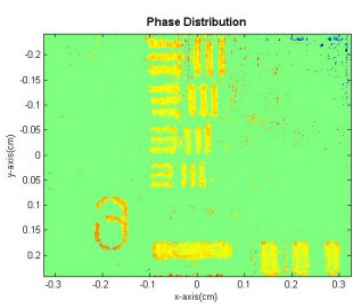

Control set (Reflection-type)

Without transparency

With transparency

(c)

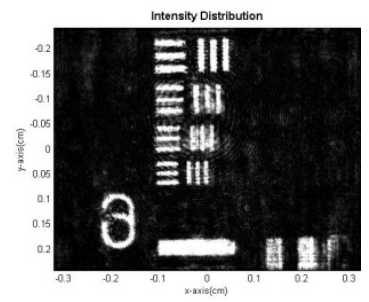

(g)

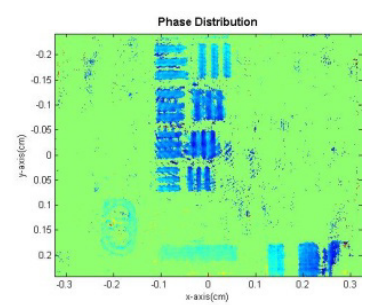

(d)

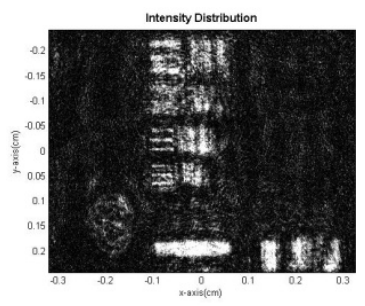

(h)

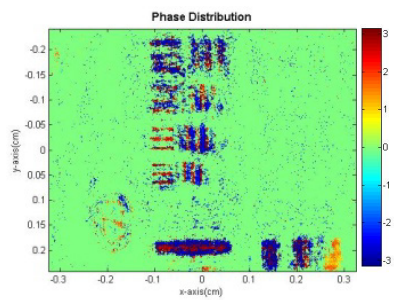

Fig. 5. (a-d) Reconstructed images of that using SPPCDHM and its control set under reflection-type configuration, without and with transparency introduced respectively, while (e-h) are corresponding phase distribution after binary mask filter.

Table 3. Threshold values to generate binary filter mask.

\begin{tabular}{|c|c|c|}
\hline \multirow{2}{*}{} & \multicolumn{2}{|c|}{ Intensity threshold value (\%) } \\
\cline { 2 - 3 } & SPPCDHM & Control Set \\
\hline Transmission-type & 31.0145 & 8.0835 \\
\hline Reflection-type & 29.3342 & 9.2751 \\
\hline
\end{tabular}

It is interesting to see that the reconstructed background intensity noises of those from PCM are greater than those from CM (much obvious from reflection-type configuration), but still having a higher level of SNR. The background noise was due to the fact that the optical elements used were non-AR-coated. Nonetheless, phase conjugation via four-wave mixing has intermediate procedures of two-wave mixing within the photorefractive crystal, such that lower intensity signals (noise) will transfer energy to higher intensity signals, and gain signal amplification. The respective theories are well developed [27, 28]. Therefore, even though the noises may not be fully compensated within the uncertainty of control, the existence of noise are negligible. Apparently, the gap between signal and noise has increased, which gave us a higher threshold value to discriminate associated noise from the retrieved signals. By employing PCM for noise suppression, the ability in discriminating noise has gained 3.5486 fold on average.

Secondly, the reconstructed images of both control set was not as good as that in common situation. This is because physical compensating technique was adopted to compensate the quadratic phase term. The virtual offset lens technique [25] offers a simple and effective approach for the phase compensation. Nevertheless, to have a retro-reflection at the focal point of a lens, wavefront is prone to aberrations, which broke down the nice symmetry that was intended to be. Therefore, the quadratic phase term is not fully compensated. Thus we can note the presence of a circular dark fringe around the edges of the image. In addition, the quadratic phase term will serve as a chirp function to blur the images. In contrast to the scenario encountered by using CM, PCM gives clear and sharp images. As mentioned in previous section, the idea of perturbed plane waves can be extended to arbitrary wavefront, and was proven in real practice. Without loss of generality, such property will also relax the stringent requirement for physical alignment.

It should be clear that the results have showed the capabilities of improvement in image fidelity, by utilizing a PCM to suppress noise prior to recording stage. However, SPPCDHM technique still has spaces for improvement. The experiment was done under ambient environment of $27.5^{\circ} \mathrm{C}$ and $40 \%$ humidity, $50^{\circ}$ incident angle with $94.3 \mu \mathrm{W}$ effective input into the PCM (0.10W laser output), and a steady-state phase-conjugated signal forms within minutes. Hence, it is now an open question to the communities in phase conjugation to reintroduce phase conjugation techniques into DHM, whether by utilizing different crystals or have different dopants, or even employ mutually-pumped scheme, in order to enhance the reflectivity or to boost the respond time. A variety of choices of media is available in producing phase-conjugated signals, in response to different domain of wavelength per area of interest [29].

\section{Conclusion}

To conclude this research, we have adopted SPPC technique into DHM for scattering noise suppression subjected to the object arm. The object signal was back propagated via a PCM to retrace the original form of the object signal, suppressing scattering noises and also 
compensating aberrations prior to the recording stage. On pure physical compensation basis, the noise level has decreased 2.9096 times on average, and the ability to discriminate noise from the signal of interest has increased 3.5486 fold on average. Under the architect of SPPCDHM by using a 20x objective lens, a net magnification power of $\sim 29 \mathrm{x}$ and resolvability of $2.19 \mu \mathrm{m}$ (6 element of group 7, USAF 1951 resolution test target) was achieved.

\section{Acknowledgments}

The authors thank Ministry of Science and Technology (MOST), Taiwan for financial support (103-2221-E-451006) and (104-2221-E-451-011). The authors thank the reviewers in pointing out some concerns related to reconstructed images, in turn made the outcome of this paper to be better than expected.

\section{References}

1. U. Schnars and W. Jüptner, "Direct recording of holograms by a CCD target and numerical reconstruction," Appl Opt. 33(2), 179-181 (1994).

2. E. Cuche, P. Marquet, and C. Depeursinge, "Spatial filtering for zero-order and twin-image elimination in digital off-axis holography," Appl. Opt. 39(23), 4070-4075 (2000).

3. N. Demoli, J. Meštrović, and I. Sović, "Subtraction digital holography," Appl. Opt. 42(5), 798-804 (2003).

4. T. Zhang and I. Yamaguchi, "Three-dimensional microscopy with phase-shifting digital holography," Opt. Lett. 23(15), 1221-1223 (1998).

5. J. P. Liu and T. C. Poon, "Two-Step-Only Quadrature Phase-Shifting Digital Holography," Opt. Lett. 34(3) 250-252 (2009).

6. G. L. Chen, C. Y. Lin, H. F. Yau, M. K. Kuo, and C. C. Chang, "Wavefront reconstruction without twin-image blurring by two arbitrary step digital holograms," Opt. Express 15(18), 11601-11607 (2007)

7. Y. L. Lee, Y. C. Lin, H. Y. Tu, and C. J. Cheng, "Phase measurement accuracy in digital holographic microscopy using a wavelength-stabilized bser diode," J. Opt. 15(2), 025403 (2013).

8. F. Charriére, B. Rappaz, J. Kühn, T. Colomb, P. Marquet, and C. Depeursinge, "Influence of shot noise on phase measurement accuracy in digital holographic microscopy," Opt. Express 15(14), 8818-8831 (2007).

9. J. C. Mauro, S. Raghavan, and A. B. Ruffin, "Enhanced stimulated Brillouin scattering threshold through phase control of multitone phase modulation," Opt. Eng. 49(10), 100501-1-100501-3 (2010).

$10 . \quad J$. S. Jeong, H. S. Kim, B. S. Choi, D. C. Kim, K. S. Kim, M. R. Park, and O. K. Kwon, "Mitigation of Rayleigh crosstalk using noise suppression technique in 10-Gb/s REAM-SOA," Opt. Express 20(24), 26373- 26378 (2012).

11. H. Guillet de Chatellus, I. Moldovan, V. Fesquet, and J. P. Pique, "Suppression of Rayleigh scattering noise in sodium laser guide stars by hyperfine depolarization of fluorescence," Opt. Express 14(24), 11494-11505 (2006).

12. J. Réhault and J. Helbing, "Angle determination and scattering suppression in polarization-enhanced two-dimensional infrared spectroscopy in the pump-probe geometry," Opt. Express 20(19), 21665- 21677 (2012).

13. B. Cochenour, S. O'Connor, and L. Mullen, "Suppression of forward-scattered light using high-frequency intensity modulation," Opt. Eng. 53(5), 051406-1-051406-8 (2014).

14. O. Okusaga, J. P. Cahill, A. Docherty, C. R. Menyuk, W. Zhou, and G. M. Carter, "Suppression of Rayleigh-scatteringinduced noise in OEOs," Opt. Express 21(19), 22255-22262 (2013).

15. A. Moussaïd and P. N. Pusey, "Multiple scattering suppression in static light scattering by cross-correlation spectroscopy," Phys. Rev. E 60(5), 5670-5676 (1999)

16. M. Cui, and C. Yang, "Implementation of a digital optical phase conjugation system and its application to study the robustness of turbidity suppression by phase conjugation," Opt. Express 18(4), 3444-3455 (2010).

17. T. R. Hillman, T. Yamauchi, W. Choi, R. R. Dasari, M. S. Feld, Y. K. Park, and Z. Yaqoob, "Digital optical phase conjugation for delivering two-dimensional images through turbid media," Scientific Reports 3(1909), 1-5 (2013).

18. Z. Yaqoob, D. Psaltis, M. S. Feld, and C. Yang, "Optical phase conjugation for turbidity suppression in biological samples," Nature Photon. 2(2), 110-115 (2008).

19. E. J. McDowell, M. Cui, I. M. Vellekoop, V. Senekerimyan, Z Yaqoob, and C. Yang, "Turbidity suppression from the ballistic to the diffusive regime in biobgical tissues using optical phase conjugation," J. Biomed. Opt. 15(2), 025004-1-025004-11 (2010).

20. M. Cronin-Gobmb, B. Fischer, J. O. White, and A. Yariv, "Theory and Applications of Four-Wave Mixing in Photorefractive Media," IEEE J. Quantum Electron. 20(1), 12-30 (1984).

21. C. C. Chang and D. R. Selviah, "Mutually pumped phase-conjugate mirror: fish-head configuration," Opt. Lett. 20(7), 677-679 (1995).

22. C. C. Chang, H. F. Yau, N. J. Cheng, and P. X. Ye, "Pentagon-shaped $0^{\circ}$-cut $\mathrm{BaTiO}_{3}$ as an efficient self-pumped phase conjugator," Appl Opt. 38(35), 7206-7213 (1999).

23. M. Zgonik, P. Bernasconi, M. Duelli, R. Schlesser, P. Günter, M. H. Garrett, D. Rytz, Y. Zhu, and X. Wu, "Dielectric, elastic, piezoelectric, electro-optic, and elasto-optic tensors of $\mathrm{BaTiO}_{3}$ crystals," Phys. Rev. B 50(9), 5941-5949 (1994).

24. J. Feinberg, "Self-pumped, continuous-wave phase conjugator using internal reflection," Opt. Lett. 7(10), 486-488 (1982).

25. Y. K. Chew, M. T. Shiu, J. C. Wang, and C. C. Chang, "Compensation of phase aberration by using a virtual confocal scheme in digital hobgraphic microscopy," Appl. Opt. 53(27), G184-G191 (2014).

26. C. C. Chang, M. T. Shiu, J. C. Wang, C. H. Wu, and Y. K. Chew, "Disturbance-free digital holographic microscopy via a micro-phase-step approach," Opt. Lasers Eng. 68, 166-171 (2015).

27. Y. Fainman, E. Klancnik, and Sing H. Lee, "Optimal coherent image amplification by two-wave coupling in photorefractive $\mathrm{BaTiO}_{3}$," Opt. Eng. 25(2), 228-234 (1986).

28. N. Koukourakis, T. Abdelwahab, M. Y. Li, H. Höpfner, Y. W. Lai, E. Darakis, C. Brenner, N. C. Gerhardt, and M. R. Hofmann, "Photorefractive two-wave mixing for image amplification in digital holography," Opt. Express. 19(22), 22004-22023 (2011).

29. P. Günter, J. P. Huignard, Photorefractive Materials and Their Applications 2 (Springer 2007). 